\title{
EXISTENCIA GLOBAL Y DECAIMIENTO DE LA ENERGÍA DE UNA ECUACIÓN DE KIRCHOFF CON DISIPACIÓN LOCALIZADA
}

Eugenio Cabanillas L., Zacarias Huaringa y Juan Bernui B ${ }^{1}$.

RESUMEN.- En este trabajo estudiamos la existencia y unicidad de la solución global de la ecuación de Kirchoff

$$
u^{\prime \prime}-M\left(\int_{\Omega}|\nabla u|^{2} d x\right) \Delta u+a(x) u^{\prime}=0
$$

Con una disipación au' y demostraremos el decaimiento exponencial de su energía.

\section{INTRODUCCIÓN}

Sea $\Omega$ un dominio acotado de $R^{N}$ con frontera regular $\Gamma$. Fijemos $x_{0} \in R^{N}$. Sea $a=a(x) \in L^{\infty}(\Omega)$ una función acotada no negativa tal que

$$
a(x) \geq a_{0}>0 \text { e.s en } \omega .
$$

Donde $\omega$ es una vecindad de $\Gamma_{0}$,

$$
\Gamma_{0}=\left\{x \in \Gamma:\left(x-x_{0}\right) \cdot v(x)>0\right\}
$$

$v(x)$ es el vector exterior normal unitario en $x \in \Gamma$ y $a_{0}$ es una constante positiva. Por una vecindad de $\Omega$, entenderemos a la intersección de $\Omega$ y una vecindad de $\Gamma_{0}$.

En el presente trabajo estamos interesados en demostrar la existencia y unicidad de la solución global, así como el comportamiento asintótico de la energía del problema:

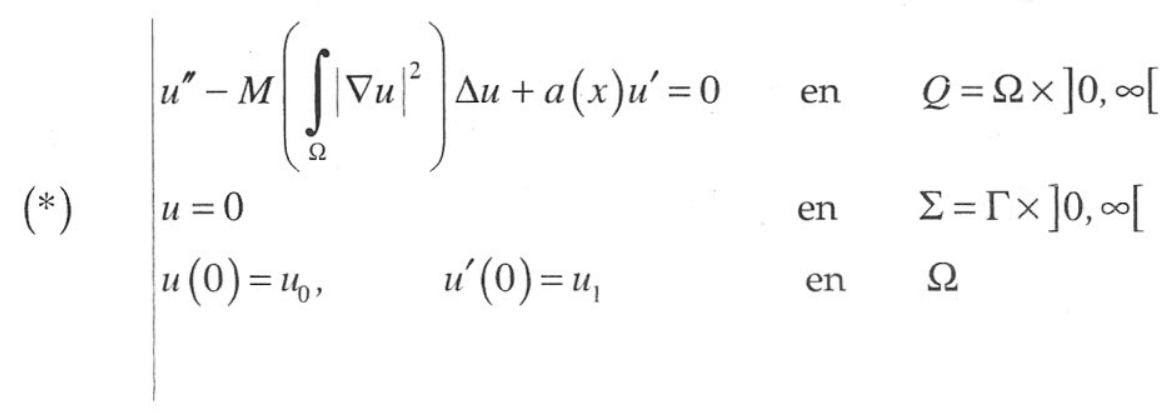

\footnotetext{
1 Universidad Nacional Mayor de San Marcos - Facultad de Ciencias Matemáticas - Instituto de Investigación.
} 
cuando $a(x)$ es efectiva en el subconjunto $\omega$ de $\Omega$.

El caso $M(x)=1$ ha sido estudiado por muchos autores: M. Nakao [9], P. Martínez [7]. E. Cabanillas - J. Bernui - Z. Huaringa [1], L.R. Tcheougue[10], E. ZuaZua [12]. Ecuaciones del Tipo Kirchoff-Carrier con disipaciones en la frontera fueron vistas por: I. Lasieccka \& J. Ong [5], M. Milla Miranda \& L.P. San, Gil Jutuca [8], y otros.

\section{PRELIMINARES}

Los espacios que usaremos son los usuales y omitiremos su definición.

Denotemos lo siguiente:

$$
\begin{aligned}
& (u, v)=\int_{\Omega} u(x) \mathrm{v}(x) d x, \quad|u|^{2}=\int_{\Omega}|u(x)|^{2} d x \\
& u^{\prime}=\frac{\partial u}{\partial t}, \quad R=\max _{x \in \Omega}\left\|x-x_{0}\right\|
\end{aligned}
$$

Las hipótesis sobre $M$ son:

$$
M \in C^{1}\left(\left[0, \infty[), \quad M(\lambda) \geq m_{0}>0, \forall \lambda \geq 0\right.\right.
$$

$$
M^{1}(\lambda) \geq 0
$$

La hipótesis sobre los datos son:

$$
u_{0} \in H_{0}^{1}(\Omega) \cap H^{2}(\Omega), u_{1} \in H_{0}^{1}(\Omega)
$$

Ahora podemos establecer nuestro resultado principal.

Teorema 2.1 Bajo las hipótesis (H.1) - (H.3), el problema $\left(^{*}\right)$ posee una única solución u tal que:

$$
\begin{aligned}
& u \in L^{\infty}\left(0, \infty ; H_{0}^{1}(\Omega) \cap H^{2}(\Omega)\right) \\
& u^{\prime} \in L^{\infty}\left(0, \infty ; H_{0}^{1}(\Omega)\right) \\
& u^{\prime \prime} \in L^{\infty}\left(0, \infty ; L^{2}(\Omega)\right)
\end{aligned}
$$

Definiendo la energía asociada al sistema ${ }^{*}$ ) como

$$
E(t)=\frac{1}{2}\left|u^{\prime}(t)\right|^{2}+\frac{1}{2} \widehat{M}\left(|\nabla u(t)|^{2}\right)
$$

donde $\hat{M}(\lambda)=\int_{0}^{\lambda} M(s) d s$; existen constantes positivas $C_{0}$ y $\gamma$ tales que: 


$$
E(t) \leq C_{0} e^{-\gamma t}, \forall t \geq 0
$$

Para obtener (2.5) usamos el siguiente,

Lema 2.2 (Haraux [3]). Sea $E:[0, \infty[\rightarrow[0, \infty$ [ una función no creciente localmente absolutamente continua, tal que, existen constantes positivas $\beta$ y A con,

$$
\int_{S}^{\infty} E(t)^{\beta+1} d t \leq A E(S), \forall S>0
$$

Entonces

$$
E(t) \leq\left\{\begin{array}{l}
E(0) e^{1-\frac{1}{A}}, \forall t \geq 0, \text { si } \beta=0 \\
{\left[A\left(1+\frac{1}{\beta}\right)\right]^{\frac{1}{\beta} t^{\frac{1}{\beta}}}, \text { si } \beta>0}
\end{array}\right.
$$

\section{LA ECUACIÓN LINEALIZADA}

En esta sección estudiaremos la existencia y unicidad de la solución global del sistema linealizado:

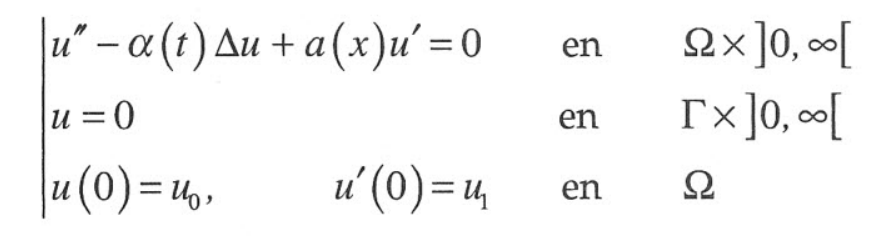

donde $u_{0}$ y $u_{1}$ satisfacen (H.3), siendo:

$$
\alpha \in C(0, T), \alpha^{\prime} \in L^{\infty}(0, T), \alpha(t) \geq m_{0}>0, \forall t \in[0, T]
$$

Teorema 3.1 Bajo las hipótesis (H.3) y (3.2) el sistema (3.1) admite una única solución $u$ tal que:

$$
\begin{aligned}
& u \in L^{\infty}\left(0, T ; H_{0}^{1}(\Omega) \cap H^{2}(\Omega)\right) \\
& u^{\prime} \in L^{\infty}\left(0, T ; H_{0}^{1}(\Omega)\right) \\
& u^{\prime \prime} \in L^{\infty}\left(0, T ; L^{2}(\Omega)\right)
\end{aligned}
$$

Demostración.- Se demuestra usando una base $\left\{\omega_{\gamma}\right\}_{\gamma \geq 1}$ de $H_{0}^{1}(\Omega) \cap H^{2}(\Omega)$, considerando el subespacio $V_{m}=\left[\omega_{1}, \omega_{2}, \ldots, \omega_{m}\right], m \in \mathbb{N}$ y se determina 


$$
u_{m}(t)=\sum_{j=1} g_{j m}(t) \omega_{j}
$$

solución del problema de Cauchy

$$
\mid \begin{aligned}
& \left(u_{m_{m}^{\prime \prime}}^{\prime \prime}(t), v\right)+\alpha(t)\left(\nabla u_{m}(t), \nabla v\right)+\left(a u_{m}^{\prime}(t), v\right)=0 \\
& u_{\cdot_{m}}(0)=u_{0 m} \rightarrow u_{0} \quad \text { en } H_{0}^{1}(\Omega) \cap H^{2}(\Omega) \\
& u_{m_{m}^{\prime}}^{\prime}(0)=u_{1 m} \rightarrow u_{1} \quad \text { en } H_{0}^{1}(\Omega)
\end{aligned}
$$

Por la teoria de las E.D.O. se prueba que existe una solución local a (3.6) en algún intervalo $\left[0, T_{m}\right]$. Esta solución será extendida por medio de estimativas a priori.

Estimativa a priori I.- Se hace $\mathrm{v}=u_{m}^{\prime}(t)$ en (3.6) y se obtiene

$$
\begin{aligned}
& \left|u_{m}^{\prime}(t)\right|^{2}+\alpha(0)\left|\nabla u_{m}(t)\right|^{2}+2 \int_{0}^{t} \int_{\Omega}\left|a^{1 / 2} u_{m}^{\prime}(s)\right|^{2} d x d s \leq \\
& \leq\left(\left|u^{\prime}(0)\right|^{2}+\alpha(0)|\nabla \mathrm{v}(0)|^{2}\right) \exp \left(2 \int_{0}^{t} \frac{\left|\alpha^{\prime}(s)\right|}{\alpha(s)} d s\right), 0 \leq t \leq T
\end{aligned}
$$

Estimativa a priori II.- Primero se acota $\left|u^{\prime \prime}(0)\right|$ considerando $t=0$ en (3.6) y $\mathrm{v}=u_{m}^{\prime \prime}(0)$ :

$$
\left|u_{m}^{\prime \prime}(0)\right| \leq \alpha(0)|\Delta u(0)|+|a|_{\infty}\left|u^{\prime}(0)\right|
$$

Ahora derivando (3.6) respecto a $t$ y considerando $\mathrm{v}=u_{m}^{\prime \prime}(t)$ resulta:

$$
\begin{aligned}
& \left|u_{m}^{\prime \prime}(t)\right|^{2}+\alpha(t)\left|\nabla u_{m}^{\prime}(t)\right|^{2}+\frac{3}{2} \int_{0}^{t} \int_{\Omega}\left|a^{\frac{1}{2}} u_{m}^{\prime \prime}(s)\right|^{2} d x d s \leq \\
& \leq I_{0}(t) \exp \left(2 \int_{0}^{t} \frac{\left|\alpha^{\prime}(s)\right|}{\alpha(s)} d s\right)
\end{aligned}
$$

donde $I_{0}(t)=\left(\alpha(0)|\Delta u(0)|+|a|_{\infty}\left|u^{\prime}(0)\right|\right)^{2}+\alpha(0)|\nabla u(0)|^{2}+$

$$
+\left[\frac{\left|\alpha^{\prime}\right|_{\infty}}{m_{0}}\left(\left|u^{\prime}(0)\right|^{2}+\alpha(0)|\nabla u(0)|^{2}\right)\right]^{2} \exp \left(2 \int_{0}^{t} \frac{\left|\alpha^{\prime}(s)\right|}{\alpha(s)} d s\right)
$$


Estimativa a priori III.- En (3.6) haciendo $\mathrm{v}=\Delta u_{m}(t)$ resulta:

$$
\begin{aligned}
\left|\Delta u_{m}(t)\right| \leq & \frac{|\alpha|_{\infty}}{m_{0}}\left(\left|u^{\prime}(0)\right|^{2}+\alpha(0)|\nabla u(0)|^{2}\right)^{\frac{1}{2}} \exp \left(\int_{0}^{t} \frac{\left|\alpha^{\prime}(s)\right|}{\alpha(s)} d s\right)+ \\
& +\frac{l_{0}(t)^{\frac{1}{2}}}{m_{0}} \exp \left(\int_{0}^{t} \frac{\left|\alpha^{\prime}(s)\right|}{\alpha(s)} d s\right)
\end{aligned}
$$

De las estimativas I, II y III, existe una subsucesión, denotada por $\left(u_{m}\right)_{m \geq 1}$, de $\left(u_{m}\right)_{m \geq 1}$ tal que

$$
\begin{aligned}
& u_{m} \rightarrow u \text { débil } \quad * \text { en } \quad L^{\infty}\left(0, T ; H_{0}^{1}(\Omega) \cap H^{2}(\Omega)\right) \\
& u_{m}^{\prime} \rightarrow u^{\prime} \text { débil } \quad * \text { en } \quad L^{\infty}\left(0, T ; H_{0}^{1}(\Omega)\right) \\
& u_{m}^{\prime \prime} \rightarrow u^{\prime \prime} \text { débil } \quad * \text { en } \quad L^{\infty}\left(0, T ; L^{2}(\Omega)\right)
\end{aligned}
$$

De (3.12) y (3.13) $u_{m}^{\prime} \rightarrow u^{\prime}$ fuerte en $L^{2}\left(0, T ; L^{2}(\Omega)\right)$, lo que implica:

$$
a u_{m}^{\prime} \rightarrow a u^{\prime} \text { débil } * \text { en } L^{\alpha}\left(0, T ; L^{2}(\Omega)\right)
$$

Las convergencias (3.11) - (3.14) permiten pasar al límite en (3.6).

No es difícil verificar la unicidad.

\section{SOLUCIONES LOCALES Y GLOBALES}

En esta sección probaremos que el problema $\left(^{*}\right)$ posee una solución en algún intervalo $\left[0, T_{m}\right]$ haciendo uso del teorema del punto fijo. Más aún mostramos que esta solución es única.

Para $T>0$ y $R>0$ definimos el espacio de soluciones como

$$
X_{T, R}=\left\{\begin{array}{l}
u \in L^{\infty}\left(0, T ; H_{0}^{1}(\Omega) \cap H^{2}(\Omega)\right), u \in L^{\infty}\left(0, T ; H_{0}^{1}(\Omega)\right) \cap C\left(0, T, L^{2}(\Omega)\right), \\
|u|_{L^{\infty}\left(0, T, H_{0}^{1} \cap H^{2}\right)}^{2}+\left|u^{\prime}\right|_{L^{\infty}\left(0, T, H_{0}^{1}\right)}^{2} \leq R^{2}, \text { en }[0, T], u(0)=u_{0}, u^{\prime}(0)=u_{1}
\end{array}\right\}
$$

Es fácil verificar que $X_{T, R}$ con la métrica

$$
d(u, v)=|u-v|_{L^{\infty}\left(0, T, H_{0}^{1}\right)}^{2}+\left|u^{\prime}-v^{\prime}\right|_{C\left(0, T ; L^{2}(\Omega)\right)}^{2}
$$

es un espacio métrico completo.

Definimos una aplicación no líneal $S$ de la siguiente manera. 
Para $\mathbf{v} \in X_{T, R}, u=S \mathbf{v}$ es la única solución de la ecuación líneal.

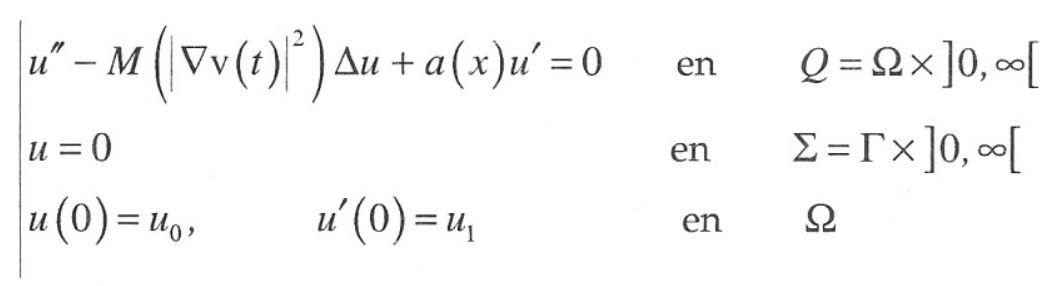

Mostramos que existe $T>0$ y $R>0$ tal que $S\left(X_{T, R}\right) \subseteq X_{T, R}$

Sea $\alpha(t)=M\left(|\nabla \mathbf{v}(t)|^{2}\right)$ y $\kappa=\max \left\{M^{1}(\lambda), 0 \leq \lambda \leq R^{2}\right\}$

entonces:

$$
\left|\alpha^{\prime}(t)\right| \leq M^{1}\left(|\nabla \mathbf{v}(t)|^{2}\right)\left|2\left(\nabla \mathbf{v}(t), \nabla \mathbf{v}^{\prime}(t)\right)\right| \leq K R^{2}
$$

ahora de (3.9) y (3.11)

$$
\begin{gathered}
\left|\nabla u^{\prime}(t)\right|^{2} \leq \frac{1}{m_{0}} I_{0}(t) \exp \left(2 \int_{0}^{t} \frac{\left|\alpha^{\prime}(s)\right|}{\alpha(s)} d s\right) \\
|\Delta u(t)|^{2} \leq \frac{2}{m_{0}^{2}}\left[|a|_{\infty}^{2}\left(\left|u^{\prime}(0)\right|^{2}+\alpha(0)|\nabla u(0)|^{2}\right)+I_{0}(t)\right] \\
\cdot \exp \left(2 \int_{0}^{t} \frac{\left|\alpha^{\prime}(s)\right|}{\alpha(s)} d s\right)
\end{gathered}
$$

De (4.2), (4.3) y (4.4), podemos seleccionar $T_{0} \leq T$ de modo que:

$$
\left|\nabla u^{\prime}(t)\right|^{2}+|\Delta u(t)|^{2} \leq R^{2}, \forall t \in\left[0, T_{0}\right]
$$

También conseguimos demostrar que $S$ es una contracción estricta:

$$
d\left(S u_{1}, S u_{2}\right) \leq \theta d\left(u_{1}, u_{2}\right), 0<\theta<1
$$

para algún $T_{0}$ suficientemente pequeño.

Eligiendo $T_{0}$ que satisfaga (4.5) y (4.6) simultaneamente; deducimos que $S$ tiene un único punto fijo $u$. Esta función es justamente la solución buscada.

A fin de exterder la solución $u$ a todo el intervalo $[0, \infty[$, consideramos la ecuación linealizada: 


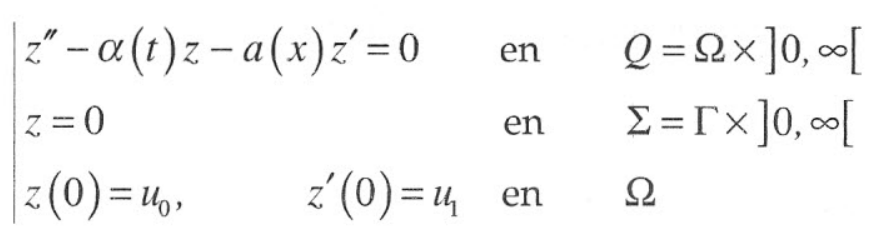

Con $\alpha(t)=M\left(|\nabla u(t)|^{2}\right), t \in\left[0, T_{0}\right]$ donde $u$ es la solución obtenida mediante el método del punto fijo.

Como $u \in X_{T_{0, R}}$ se sigue que $\alpha$ sastiface las condiciones del Teorema $3.1 \mathrm{y}$ asi:

$$
z \in C_{\omega}\left(0, T_{0}, H_{0}^{1} \cap H^{2}\right) \cap C_{\omega}^{1}\left(0, T_{0}, H_{0}^{1}(\Omega)\right) \cap C_{\omega}^{2}\left(0, T_{0}, L^{2}(\Omega)\right)
$$

Como $u$ es solución de (4.7) y este problema tiene unicidad entonces $z=u$, por lo que $u$ sastiface (4.8).

El lema de Zorn nos permite concluir que $\left(^{*}\right)$ posee una única solución maximal $u:] 0, T_{0}\left[\rightarrow H_{0}^{1}(\Omega) \cap H^{2}(\Omega)\right.$ con la regularidad (4.8).

El siguiente lema es decisivo en la demostración del teorema.

Lema 4.1 Sea u solución de $\left(^{*}\right)$ en $\left[0, T_{m}[\right.$. Si

$$
\underset{t \rightarrow T_{m}}{\limsup }\left[|\Delta u(t)|^{2}+\left|\nabla u^{\prime}(t)\right|^{2}\right]<+\infty \text { entonces } T_{m}=+\infty
$$

\section{Demostración.- Ver [11] .}

Con este lema estamos habilitados para extender la solución de $\left(^{*}\right)$ al intervalo $\left[0,+\infty\left[\right.\right.$, lo que concluye la prueba de la existencia global de la solución $u$ de $\left(^{*}\right)$.

\section{COMPORTAMIENTO ASINTÓTICO DE LA ENERGÍA}

Verifiquemos primero que $\left(^{*}\right)$ es un problema disipativo.

\section{Lema 5.1}

$$
\forall 0 \leq S<T<\infty: E(t)-E(S)=-\int_{S}^{T} \int_{\Omega} a\left(u^{\prime}\right)^{2} d x d t
$$

Demostración.- Basta multiplicar por $u^{\prime}$ la ecuación (*) e integrar por partes en $\Omega \times[S, T]$. 
Lema 5.2 Sea $q \in\left[W^{1, \infty}(\Omega)\right]^{N}, \alpha \in R$ y $\xi \in W^{1, \infty}(\Omega)$. Se verifican las siguientes identidades:

$$
\begin{aligned}
\int_{S}^{T} \int_{\Omega} M\left(|\nabla u(t)|^{2}\right) q \cdot v\left|\frac{\partial u}{\partial v}\right|^{2} d \Gamma d t=\left.(u, 2 q \cdot \nabla u+\alpha u)\right|_{S} ^{T}+ \\
+\int_{S}^{T} \int_{\Omega}\left[\left[(\operatorname{div} q-\alpha)\left[\left|u^{\prime}\right|^{2}-M\left(|\nabla u(t)|^{2}\right)|\nabla u(t)|^{2}\right] d x d t+\right.\right. \\
+2 \int_{S}^{T} \int_{\Omega} M\left(|\nabla u(t)|^{2}\right) \frac{\partial q_{k}}{\partial x_{i}} \frac{\partial u}{\partial x_{k}} \frac{\partial u}{\partial x_{i}} d x d t+ \\
+\int_{S}^{T} \int_{\Omega} a u^{\prime}(2 q \cdot \nabla u+\alpha u) d x d t \\
\left.\left(u^{\prime}, \xi u\right)\right|_{S} ^{T}+\int_{S}^{T} \int_{\Omega} \xi\left[M\left(|\nabla u|^{2}\right)|\nabla u|^{2}-\left|u^{\prime}\right|^{2}\right] d x d t+ \\
+\int_{S}^{T} \int_{\Omega} M\left(|\nabla u|^{2}\right)(\nabla u, \nabla \xi) d x d t+\int_{S}^{T} \int_{\Omega} a u^{\prime} \xi u d x d t=0
\end{aligned}
$$

La demostración del Lema 5.2 se basa en técnicas conocidas de multiplicadores. El lector interesado puede leer Lions [6] ó Komornik [4].

Para probar procedemos en dos etapas.

Etapa 1.- Aplicamos (5.2) con $q(x)=m(x), \alpha=N-1$ obtenemos

$$
\begin{aligned}
& \int_{S}^{T} \int_{\Omega}\left[|\nabla u|^{2}+M\left(|\nabla u|^{2}\right)-|\nabla u|^{2}\right] d x d t=\int_{S}^{T} \int_{\Gamma} M|\nabla u|^{2} m \cdot v\left|\frac{\partial u}{\partial v}\right|^{2}- \\
& -\left.\left(u^{\prime}, 2 m \cdot \nabla u+(N-1) u\right)\right|_{S} ^{T}-\int_{S}^{T} \int_{\Omega} a u^{\prime}[2 m \nabla u+(N-1) u] d x d t
\end{aligned}
$$

Observemos que como $u \in L^{\infty}\left(0, \Gamma, H_{0}^{1}(\Omega) \cap H^{2}(\Omega)\right)$ :

$$
m_{0} \leq M\left(|\nabla u(t)|^{2}\right) \leq m_{1} \quad, \quad \forall t \geq 0
$$

por lo que:

$$
m_{0}|\nabla u(t)|^{2} \leq \widehat{M}\left(|\nabla u(t)|^{2}\right) \leq \frac{m_{1}}{m_{0}} M\left(|\nabla u(t)|^{2}\right)|\nabla u(t)|^{2}, \forall t \geq 0 .
$$


Denotemos con

$$
E_{0}(t)=\frac{1}{2}\left|u^{\prime}(t)\right|^{2}+\frac{1}{2} M\left(\left|\nabla \mathrm{u}^{\prime}(t)\right|^{2}\right)|\nabla \mathrm{u}(t)|^{2}
$$

Luego existen constantes positivas $C_{0}$ y $C_{1}$ tales que:

$$
C_{0} E(t) \leq E_{0}(t) \leq C_{1} E(t)
$$

En lo que sigue de este trabajo todas las constantes positivas serán denotadas con C y cambiarán de línea en línea.

Acotando convenientemente en (5.4) obtenemos

$$
\int_{S}^{T} E_{0}(t) \leq C E_{0}(S)+m, R \int_{S}^{T} \int_{\Gamma_{0}}\left|\frac{\partial u}{\partial v}\right|^{2} d \Gamma d t
$$

Etapa 2.- Para estimar el último término en (5.8) usamos (5.3) con $\xi=\eta$ donde $\eta \in W^{1, \infty}(\Omega)$ es una función tal que:

$$
\mid \begin{aligned}
& 0 \leq \eta \leq 1 \\
& \eta=1 \quad \text { en } \quad \widehat{\omega} \quad ; \quad \frac{|\nabla \eta|^{2}}{\eta} \in L^{\infty}(\omega) \\
& \eta=0 \quad \text { en } \quad \frac{\Omega}{\omega}
\end{aligned}
$$

y $\hat{\omega}$ es un conjunto abierto en $\Omega$ con $\Gamma_{0} \subseteq \hat{\omega} \nsubseteq \omega$, y así obtenemos la acotación.

$$
\begin{aligned}
\frac{1}{2} \int_{S}^{T} \int_{\Omega} \eta|\nabla u|^{2} d x d t & \leq C\left[E_{0}(S)+\int_{S}^{T} \int_{\omega}\left|u^{\prime}\right|^{2}+|u|^{2} d x d t\right]+ \\
& +\varepsilon \int_{S}^{T} E_{0}(t) d t, \quad \varepsilon>0 .
\end{aligned}
$$

Ahora tomamos un vector $h \in\left[W^{1, \infty}(\Omega)\right]^{N}$ tal que,

$$
h=v \text { en } T_{0}, h \cdot v \geq 0 \text { en } \Gamma \text { y } h=0 \text { en } \Omega \backslash \hat{\omega} \text {. }
$$

Elegimos $\alpha=0 \quad$ y $q=h$ en (5.2) y obtenemos el estimado: 


$$
\begin{aligned}
\int_{S}^{T} \int_{\Gamma_{0}}\left(\frac{\partial u}{\partial v}\right)^{2} d \Gamma d t & \leq C\left[E_{0}(S)+\int_{S}^{T} \int_{\omega}\left[\left|u^{\prime}\right|^{2}+|u|^{2}\right] d x d t\right] \\
& +\delta \int_{S}^{T} E_{0}(t) d t, \quad \delta>0
\end{aligned}
$$

Combinando (5.9) y (5.10)

$$
\begin{aligned}
\int_{S}^{T} \int_{\Gamma_{0}}\left(\frac{\partial u}{\partial v}\right)^{2} d \Gamma d t & \leq C\left[E_{0}(S)+\int_{S}^{T} \int_{\omega}\left[\left|u^{\prime}\right|^{2}+|u|^{2}\right] d x d t\right]+ \\
& +\varepsilon \int_{S}^{T} E_{0}(t) d t, \quad \varepsilon>0
\end{aligned}
$$

Luego de (5.7), (5.8) y (5.11)

$$
\int_{S}^{T} E_{0}(t) d t \leq C\left[E_{0}(S)+\int_{S}^{T} \int_{\omega}\left[\left|u^{\prime}\right|^{2}+|u|^{2}\right] d x d t\right]
$$

Para absorver el último término en (5.12) adaptamos un método introducido por Conrad - Rao [2], considerando $z(t) \in H_{0}^{1}(\Omega)$ solución de:

$$
\mid \begin{array}{lll}
-\Delta z=\chi(\omega) u & \text { en } & \Omega \\
z=0 & \text { en } & \Gamma
\end{array}
$$

Multiplicando la ecuación en $\left(^{*}\right)$ por $z$ e integrando por partes en $\left.\Omega \times\right] S, T[$ y acotando obtenemos

$$
\begin{aligned}
\int_{S}^{T} \int_{\omega}|u|^{2} d x d t \leq & C\left[E_{0}(S)+\int_{S}^{T} \int_{\omega}\left|u^{\prime}\right|^{2} d x d t\right]+ \\
& +\delta \int_{S}^{T} E_{0}(t) d t, \quad \delta>0
\end{aligned}
$$

De (5.13) en (5.12) resulta:

$$
\int_{S}^{T} E_{0}(t) d t \leq C E_{0}(S)
$$

Del Lema 2.2 y la expresión (5.7) se concluye la demostración del Teorema 2.1. 


\section{BIBLIOGRAFÍA}

[1] Cabanillas, L.E. - Bernui, B.J. - Huaringa, S.Z. Energy Decay of a Linear Hyperbolic Equation with Locally Distributed Damping.- 55 S.B.A. Uberlandia 2002 - Brasil.

[2] Conrad, F. \& Rao, B. Decay of Solutions of Wave Equations in a Star - Shaped Domain with non-linear boundary feed back. Asympotic Analysis, 7(1993).

[3] Haraux, A. Semigroupes Linéaires et équations d'evolution linéaires. Periodique publications du laboratoire d'Analyse Numérique Université Pierre et Manie Curie, Paris (1978).

[4] Komornik, V. Exact Controllability and Stabilization, the Multiplier Method. RAM. Masson \& John Wiley Paris. 1994.

[5] Lasieka, I. and Ong, J. Solvability and Uniform Decays of Solutions to Quasilinear Equation with Nonlinear Boundary Dissipation. Comm in Partial Diff Eq., 24 (1999), 2069 - 2107.

[6] Lions, J. L. Contrólabite Exacte, Perturbations et stabilisation des Systémes distribués, $\mathrm{N}^{\circ} 1, \mathrm{RMA}$, Masson. Paris. (1988).

[7] Martínez, P.A. New Method to obtain Decay Rate estimates for dissipative systems with localized damping. Revista Mat. Complutense Vol. 12, No 1, (1999), $251-283$.

[8] Milla Miranda, M.S., San Gil Jutuca, L.P. Existence and Boundary Stability of Solutions for the Kirchoff Equations. Comm. in Partial Diff. Eq. 24 (1999), $1759-1800$.

[9] Nakao, M. Decay of Solutions of the Wave Equations with a Local Degenerate dissipation. Israel J. Math, 95 (1996), 25 - 42.

[10] Tcheoque Tebou, L.R. On the Decay Estimates for the Wave Equation with a Local Degenerate or Nondegenerate Dissipation. Portugal Math. Vol. 55 Fasc. 3 - (1988) 293 - 306.

[11] Tucsnak, M. Boundary Stabilization for the Stretched String Equation. Diff. Integral Equations 6(1993), 925 - 935.

[10] Zua-Zua, E. Exponential Decay for the Semilinear Wave Equation with Locally Distributed Damping. Comm. in P.D.E., 15 (1990), 205 - 235. 